\title{
THE ASTROMETRIC IMAGING TELESCOPE: NEAR-TERM DISCOVERY AND STUDY OF OTHER PLANETARY SYSTEMS
}

\author{
EUGENE H. LEVY \\ Lunar and Planetary Laboratory, University of Arizona, Tucson, AZ, USA \\ GEORGE D. GATEWOOD \\ Allegheny Observatory, University of Pittsburgh, Pittsburgh, PA. U.S.A. \\ and \\ RICHARD J. TERRILE \\ Jet Propulsion Laboratory, Pasadena, CA, USA
}

\begin{abstract}
The Astrometric Imaging Telescope (AIT) is a space-based 1.5 to 2 meter diameter telescope designed to discover and study planetary systems around other stars. The measurement objectives and instrument design aim at a definitive search for other planetary systems. The science program includes two planetary-system investigations, and the telescope carries two separate instruments: an astrometric instrument to measure the reflex motions of stars caused by the motions of unseen planets and an imaging coronagraph to directly image planets and other circumstellar matter.

The astrometric techniques is based on the concept of the Multichannel Astrometric Photometer (MAP) which passes a Ronchi ruling over a field of stars to measure the centroid position of the stars in two orthogonal coordinates. Outside of Earth's atmosphere this instrument is designed to be about two orders of magnitude more accurate than the best existing ground-based astrometric instrument, which uses the same measurement technique. The astrometric design accuracy is about 10 microseconds. This will allow detection and study of Uranus-size of larger planets in jovian orbits around several hundred nearby stars. The parallactic study of the parent star of a planetary system will accurately reveal its distance; the star's departure from inertial motion will reveal the masses of individual planets and their orbits. The periods of the orbits are obtained from analysis of the motions of the central star. With sufficient precision and time, analysis of a central star's reflex motion also yields the eccentricities of the planetary orbits. Assuming that the mass of the primary star can be accurately estimated, the measurements will also yield the masses of planets.

The imaging investigation relies on a high efficiency coronagraph to suppress diffraction wings of the bright parent star by a factor of 1000 . Laboratory tests on such a coronagraph have demonstrated a high efficiency at concentrating diffracted light into an area where it can be removed by the apodization of an image of the telescope pupil. However, in order to utilize this high efficiency, the scattered light floor of the telescope must also be a factor of 1000 below the diffraction wings. This requirement puts strong constraints on the mid-spatial frequency errors of the primary mirror. Laboratory experiments in mirror fabrication have demonstrated, with sub-scale mirrors, the required flight quality optics. Space-borne, the AIT will directly image planetary systems with the capability of detecting Jupiter-sized planets in hours around the nearby stars. Its sensitivity to faint material near bright stars exceeds that of the Hubble Space Telescope (HST) by 5 stellar magnitudes and makes the instrument ideally suited for the detection and study of the circumstellar material associated with planetary system formation.
\end{abstract}

Y. Kondo (ed.), Observatories in Earth Orbit and Beyond, 287.

(C)1990 Kluwer Academic Publishers. Printed in The Netherlands. 